AperTO - Archivio Istituzionale Open Access dell'Università di Torino

A community-built calibration system: The case study of quantification of metabolites in grape juice by qNMR spectroscopy

This is a pre print version of the following article:

Original Citation:

Availability:

This version is available http://hdl.handle.net/2318/1769159

since 2021-01-26T15:00:22Z

Published version:

DOI:10.1016/j.talanta.2020.120855

Terms of use:

Open Access

Anyone can freely access the full text of works made available as "Open Access". Works made available under a Creative Commons license can be used according to the terms and conditions of said license. Use of all other works requires consent of the right holder (author or publisher) if not exempted from copyright protection by the applicable law. 


\title{
Community-built calibration system: the case study of metabolites quantification in grape juice by qNMR spectroscopy
}

\begin{abstract}
Biagia Musio $^{a \star}$, Rosa Ragone ${ }^{a, b}$, Stefano Todisco, ${ }^{a, b}$, Antonino Rizzuti, ${ }^{a, b}$ Mario Latronico, ${ }^{a, b}$ Piero Mastrorilli, ${ }^{a, b}$ Stefania Pontrelli, ${ }^{b}$ Nicola Intini, ${ }^{b, c}$ Pasquale Scapicchio, ${ }^{d, e}$ Maurizio Triggiani, ${ }^{f}$ Tommaso Dinoia, ${ }^{f}$ Domenico Acquotti, ${ }^{g}$ Cristina Airoldi, ${ }^{\mathrm{h}}$ Michael Assfalg, ${ }^{\mathrm{i}}$ Alessandro Barge, ${ }^{\mathrm{j}}$ Francesca Benevelli, ${ }^{\mathrm{k}, \mathrm{l}}$ Davide Bertelli, ${ }^{\mathrm{m}}$ Fabio Bertocchi, ${ }^{\mathrm{n}}$ Aurimas Bieliauskas, ${ }^{\circ}$ Anna Borioni, ${ }^{\mathrm{p}}$ Augusta Caligiani, ${ }^{9}$ Emanuela Callone,${ }^{r}$ Ales Čamra, ${ }^{\text {s }}$ Flaminia Cesare Marincola, ${ }^{\mathrm{t}}$ Dinesh Chalasani, ${ }^{\mathrm{u}}$ Roberto Consonni, ${ }^{\mathrm{v}}$ Paolo Dambruoso, ${ }^{\mathrm{w}}$ Silvia Davalli, ${ }^{\mathrm{x}}$ Taylor David, ${ }^{\mathrm{u}}$ Bernd Diehl, ${ }^{y}$ James Donarski, ${ }^{z}$ Ana Gil, ${ }^{a a}$ Roberto Gobetto, ${ }^{\text {ab }}$ Luca Goldoni, ac Erwann Hamon, ${ }^{\text {ad }}$ Andrea Kobrlová, ${ }^{\text {s Fran- }}$

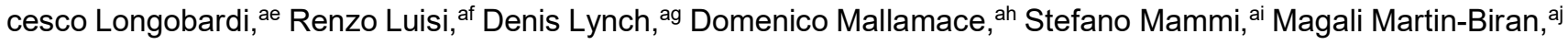
Pierluigi Mazzei, ${ }^{\text {ak }}, 1$ Andrea Mele, ${ }^{\text {al }}$ Salvatore Milone, ${ }^{\text {am }}$ Dolores Molero Vilchez, ${ }^{\text {an }}$ Roger Mulder, ${ }^{\text {ao }}$ Claudia Napoli, ${ }^{k}$ Antonella Pepe, ${ }^{\text {ap }}$ Daniele Ragno, ${ }^{\text {aq }}$ Antonio Randazzo, ${ }^{\text {ar }}$ Maria Cecilia Rossi, ${ }^{\mathrm{m}}$ Archimede Rotondo, ${ }^{\text {as,at }}$ Algirdas Sackus, ${ }^{\circ}$ Elena Sáez Barajas, ${ }^{\text {an }}$ Elisabetta Schievano, ${ }^{\text {ai }}$ Bhavaraju Sitaram, ${ }^{\mathrm{u}}$ Livio Stevanato, ${ }^{\mathrm{j}}$ Panteleimon G.Takis, ${ }^{\text {au }}$ Jan Teipel, av Freddy Thomas, ${ }^{\text {aw }}$ Elisabetta Torregiani, ${ }^{\text {ax }}$ Daniela Valensin, ${ }^{\text {ay }}$ Marina Veronesi, ${ }^{\text {ac }}$ John War-

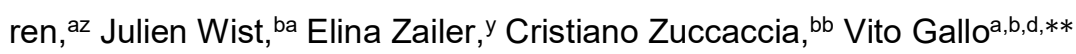

aDipartimento di Ingegneria Civile, Ambientale, del Territorio, Edile e di Chimica (DICATECh), Politecnico di Bari, via Orabona 4, I-70125, Bari, Italy

bInnovative Solutions S.r.I. - Spin Off del Politecnico di Bari, zona H 150/B, I-70015, Noci (BA), Italy

CAgenzia Regionale per la Prevenzione e la Protezione dell'Ambiente, ARPA Puglia, corso Trieste 127, I-70126, Bari, Italy dSAMER (Special Agency of the Chamber of Commerce of Bari), via E. Mola 19, I-70121, Bari, Italy eRETELAB (Italian network of the laboratories of the Chambers of Commerce) and LACHIMER (Special Agency of the Chamber of Commerce of Foggia), Via Manfredonia Km 2,200, I-71121 Foggia, Italy

fDipartimento di Ingegneria Elettrica e dell'Informazione, Politecnico di Bari, via Orabona 4, I-70125, Bari, Italy

${ }^{9}$ Centro Inter-dipartimentale Misure (CIM), Università degli Studi di Parma, Parco Area delle Scienze 23/A, I-43124, Parma, Italy hDipartimento di Biotecnologie e Bioscienze, Università of Milano-Bicocca, P.zza della Scienza 2, I-20126, Milano, Italy

'Dipartimento di Biotecnologie, Università degli Studi di Verona, Cà Vignal 1, Strada le Grazie 15, I-37134, Verona, Italy

jDipartimento di Scienza e Tecnologia del Farmaco, Università di Torino, via Verdi 8, 10124, Torino, Italy

kBruker Italia S.r.I., Viale V. Lancetti 43, I-20158, Milano, Italy

17C-Consortium for NMR Research in Biotechnology and Material Science, Via Colombo 81, I-20133, Milano, Italy"

mCentro Interdipartimentale Grandi Strumenti (CIGS), Università di Modena e Reggio Emilia, via G. Campi 213/A, 41125 Modena, Italy

nDipartimento di Scienze e Tecnologie Chimiche, Università di Roma “Tor Vergata”, via della Ricerca Scientifica, 00133 Roma, Italy

Institute of Synthetic Chemistry, Kaunas University of Technology, K. Barsausko Str. 59, LT-51423, Kaunas, Lithuania PIstituto Superiore di Sanità (ISS), Viale Regina Elena 299, I-00161, Roma, Italy

9Dipartimento di Scienze degli Alimenti e del Farmaco, Università degli Studi di Parma, Parco Area delle Scienze 23/A, I-43124, Parma, Italy

'Dipartimento di Ingegneria Industriale, via Sommarive 9, 38123 Povo (TN), Italy

${ }^{s}$ General Directorate of Customs, Budějovická 7, 14000 Prague, Czech Republic

tDipartimento di Scienze Chimiche e Geologiche, Università di Cagliari, Cittadella Universitaria di Monserrato SS 554, I-09012 Monserrato (CA), Italy 
uThe United States Pharmacopeial Convention (USP), 12601 Twinbrook Parkway, Rockville, MD 20852-1790, USA

vIstituto per lo Studio delle Macromolecole del Consiglio Nazionale delle Ricerche, (ISMAC-CNR), Laboratorio NMR, Via Bassini 15, I-20133, Milano, Italy

wIstituto per la Sintesi Organica e la Fotoreattività del Consiglio Nazionale delle Ricerche (ISOF-CNR), via P. Gobetti 101, 40129 Bologna

×Aptuit (Verona) S.r.I., Via Fleming 4, I-37135, Verona, Italy

ySpectral Service AG, Emil-Hoffmann-Straße 33, 50996 Köln, Germany

zFera Science Ltd, National Agri-Food Innovation Campus, Sand Hutton, York, YO41 1LZ, United Kingdom

${ }^{a a}$ CICECO - Aveiro Institute of Materials, Department of Chemistry, Technological Laboratories, University of Aveiro, 3810-093

Aveiro, Portugal

abDipartimento di Chimica, Università degli Studi di Torino, via Pietro Giuria 7, 10125 Torino, Italy

acFondazione Istituto Italiano di Tecnologia (IIT), via Morego 30, 16163 Genova, Italy

adAérial, 250 rue Laurent Fries - CS40443, 67412, Illkirch Cedex, France

aeDipartimento di Chimica, Università degli Studi di Bari "A. Moro", via Orabona 4, I-70125 Bari, Italy

afDepartment of Pharmacy - Drug Sciences, University of Bari "A. Moro”, FLAME-Lab - Flow Chemistry and Microreactor Technology Laboratory, Via E. Orabona 4, 70125 Bari, Italy

agAnalytical \& Biological Chemistry Research Facility (ABCRF), University College Cork, Western Road, Cork City, Ireland

ahDipartimento di Scienze Matematiche e Informatiche, Scienze Fisiche e Scienze della Terra, Università degli Studi di Messina,

Viale F. Stagno d'Alcontres 31, I-98166 Messina, Italy

aiDipartimento di Scienze Chimiche, Università degli Studi di Padova, via Marzolo 1, I-35100, Padova, Italy

ajCtre Recherche Valorisation Application (CEREVAA), 12 Allée ISAAC NEWTON, 33650 MARTILLAC, France

akUniversità di Napoli Federico II, Centro Interdipartimentale di Risonanza Magnetica Nucleare (CERMANU), via Università 100 , 80055 Portici, Italy

alDipartimento di Chimica, Materiali e Ingegneria Chimica "Giulio Natta", via Mancinelli 7, 20131 Milano, Italy

am Istituto Zooprofilattico Sperimentale dell'Abruzzo e del Molise "G. Caporale" - Food Chemistry and Residues Unit, Campo Boario, I-64100 Teramo, Italy

an Universidad Complutense de Madrid, Avda. Complutense s/n Aulario Facultad de Quimicas, 28040, Madrid, Spain

${ }^{\text {ao }}$ Commonwealth Scientific and Industrial Research Organisation, Australian Government - CSIRO, Clayton South Vic 3169 Australia

apCenter for Cancer Research Hansen Life Sciences Research, Purdue University, 720 Clinic Drive, West Lafayette, IN 479072112, Indiana.

aqDipartimento di Scienze Chimiche e Farmaceutiche, Università di Ferrara, via Fossato di Mortara 17, 44121 Ferrara, Italy

arDipartimento di Farmacia, Università di Napoli, via D. Montesano, 80131, Napoli, Italy

as Dipartimento di Scienze biomediche, odontoiatriche e delle immagini morfologiche e funzionali (BIOMORF), Università di Messina, Piazza Pugliatti 1, 98122 Messina, Italy.

atScience4life s.r.I., via Leonardo Sciascia Coop. Fede Pal.B, 98168 Messina, Italy

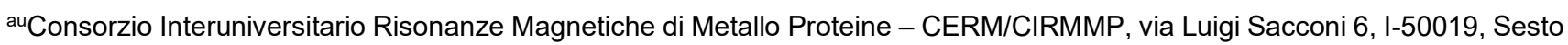

Fiorentino (FI), Italy

${ }^{\text {av }}$ Chemical and Veterinary Investigation Agency of East-Westphalia-Lippe, Westerfeldstraße 1, 32758, Detmold, Germany

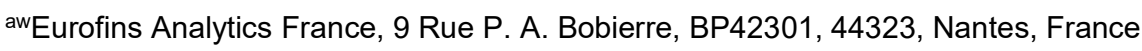

axDipartimento di Scienze Chimiche, Università di Camerino, via S. Agostino 1, 62032, Camerino, Italy

ay Dipartimento di Biotecnologie, Chimica e Farmacia, via A. Moro 2, 53100 Siena, Italy

azLGC Limited, Queen's Road, TW11 OLY, Teddington, United Kingdom

${ }^{\text {ba } C i u d a d e l a ~ U n i v e r s i t a r i a ~ M e l e n d e z, ~ U n i v e r s i d a d ~ d e l ~ V a l l e ~ C a l l e ~} 13$ No 100-00 Cali, Colombia

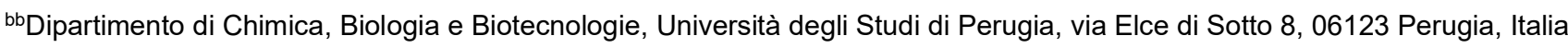
${ }^{1}$ The present address is Dipartimento di Farmacia (DIFARMA), Università degli Studi di Salerno, 84084 Fisciano (SA), Italy 
${ }^{*}$ Corresponding author.

${ }^{* *}$ Corresponding author

E-mail addresses: biagia.musio@poliba.it (B. Musio), vito.gallo@poliba.it (V. Gallo)

\author{
Keywords: \\ qNMR \\ inter-laboratory comparison \\ calibration \\ multiregression \\ validation
}

food authenticity

ABSTRACT: Nuclear Magnetic Resonance (NMR) is an analytical technique extensively used in almost every chemical laboratory for structural identification. This technique, providing statistically equivalent signals independently on the spectrometer hardware features, is advantageously employed for the traceability and quantification of analytes within food samples. Nevertheless, to date a few official related guidelines are reported. The main goal of the present study is to trace a roadmap for the data analysts to verify the reproducibility of the spectroscopic NMR data produced for a given matrix by spectrometers with different features in manufacturer and magnetic strength. Specifically, a sequence of chemometric tests was introduced and applied to the calibration data produced by 65 different spectrometers involved in an interlaboratory comparison, giving access to the development of a community-built calibration system which was employed to verify the performance of the working spectrometers and the reproducibility of the predicted concentrations. This work, describing the approach and the results of the qNMR method applied to the analysis of the grape juice as a case study, may give a boost to the introduction of qNMR protocols in standardized analytical procedures to speed up and enhance the traceability of unknown samples.

\title{
INTRODUCTION
}

Relative or absolute quantification of an analyte in a matrix consists of a sequence of operations carried out under defined and agreed methods, which are developed according to technical specificity of the analytical tool, the analyte and the matrix. Among the operations required to quantify a substance, calibration processes deserve special consideration. According to the International Bureau of Weights and Measures, calibration is defined as "Operation that, under specified conditions, in a first step, establishes a relation between the quantity values with measurement uncertainties provided by measurement standards and corresponding indications with associated measurement uncertainties (of the calibrated instrument or secondary standard) and, in a second step, uses this information to establish a relation for obtaining a measurement result from an indication".[1] Usually, a calibration curve is developed to find the optimal equation relating the response of the selected analytical technique and the concentration of a set of standard samples of the analyte with known concentration. Such equation is exploited to derive the concentration of the analyte contained in an unknown sample. The calibration conditions vary with time and instrumental use thus, depending on the specific analytical issue, a periodical update is required. Therefore, the calibration process has an important impact on both cost and execution time of the analysis.

Establishing metrological traceability is a prerequisite to obtain a reliable metrological comparability of the measurement results produced at different laboratories and at different times. As stated by De Bièvre et al., achieving metrological comparability of measurement results requires the definitions of concepts of calibration hierarchies providing metrological traceability chains, which enable the establishment of metrological traceability of measured quantity values to a common and stable metrological reference.[2] Ideally, in order to produce reliable and traced measurement results with the corresponding measurement uncertainties for a given method, the whole analytical process should be conducted by a community-built system able to simultaneously manage calibration and traceability steps for many operators. Such a system should $i$ ) collect calibration data produced by many operators, $i i$ ) process data to develop a community-built reference calibration curve and iii) provide results (exploiting the community-built reference calibration curve) to many operators after submission of data regarding unknown samples. Many advantages may derive from using such system. First, a number of community-built reference calibration curves, and, thus, reference concentration values for different analytes in different matrices can be developed. Moreover, such community-built database can be continuously updated by introduction of new calibration data, even if produced by laboratories not directly involved in the initial calibration curve building. Therefore, the reference calibration data (curve parameters and predicted analyte concentration) should become more precise and accurate and could be ultimately used as reference values to test the performance of the laboratories. The only requirement for creation of this communitybuilt system is the use of an analytical technique able to generate, for a given sample, statistically equivalent signals. In other words, any sample should be represented by the same instrumental response when analysed by different instruments.

In this context, recently, by means of inter-laboratory comparisons (ILCs), we demonstrated that NMR spectroscopy can provide statistically equivalent signals when the same sample is analysed by spectrometers that are different in terms of magnetic field strength, manufacturer, hardware configurations and age. [3,4] Indeed, the exclusive correlation between the resonance frequency of a signal and the type of nuclei associated to that signal, makes NMR spectroscopy a powerful technique for structural determination 
and quantification.[5,6] Since the intensity of a signal is linearly proportional to the number of NMR active nuclei generating the signal, the response factor (ratio between the signal produced by the analyte and the quantity of analyte which produces the signal) is independent on the molecule and the quantification of the analyte can be achieved directly by calculating the area under the signal (integral).[7] Moreover, the design of new pulse sequences for FIDs acquisition[8-13] and novel algorithms for data processing[1417] enhanced the capability of NMR for discriminating among very similar compounds contained in complex mixtures, as pharmaceutical, natural products, agrochemicals, foodstuff, and biological fluids. Nevertheless, to date few official protocols have been reported which employ NMR technique for purity assessment and quantification purposes.[18] While the experimental conditions (pulse sequence, acquisition parameters, post-processing strategy) assuring the intra-laboratory repeatability are well established,[1921] still few studies are available discussing the reproducibility assessment of qNMR data obtained when the same sample is analysed by different operators and spectrometers with variable features (manufacturer, $\mathrm{B}_{0}$ field strength).[22-25] Taking into consideration the extensive application of qNMR in different fields of chemical science, it appears as a matter of urgency to overcome this significant shortcoming and make qNMR an internationally accepted standard analytical technique.

In this paper, with the aim to explore the possibility to create a community-built quantification system, we report on the critical aspects which often affect the interlaboratory reproducibility during the quantitative analysis of components contained in complex mixtures via qNMR. Specifically, exploiting the big amount of spectroscopic data produced during an interlaboratory comparison involving 65 spectrometers, the concentrations of four selected metabolites (alanine, arginine, glucose, and fructose) contained in the grape juice ( $c v$. Primitivo) are predicted via calibration lines developed by standard addition method. A sequence of appropriate chemometric tests (Figure 1, model assessment) are applied to the predictive models developed individually by the ILC participants. Upon consideration of the likely sources of error, we establish a strategy for assessing the performance of the laboratories during the different stages of the quantitative analysis. The well-performing models are tested to predict the unknown concentrations of the metabolites contained in a test sample and the obtained data are evaluated in terms of reproducibility, allowing to identify and validate the statistical equivalent signals in the NMR spectrum (figure 1, prediction assessment).

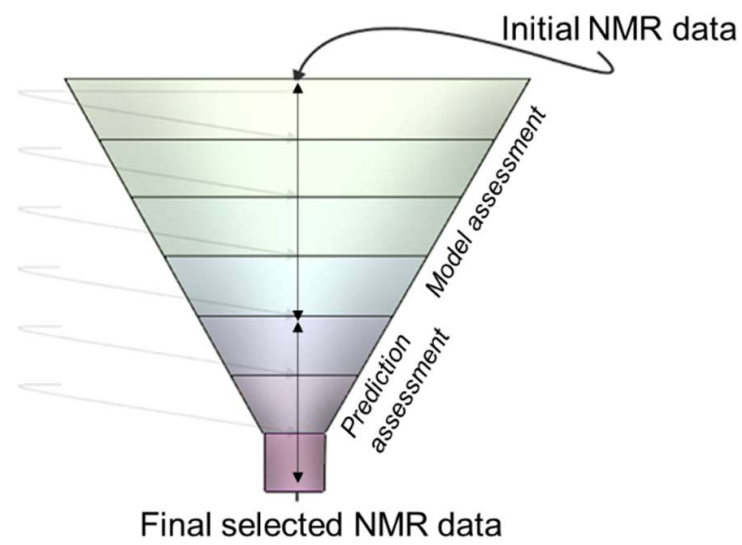

Figure 1. Schematic representation of the selection process for the evaluation of the prediction models designed by 65 ILC participants.

\section{EXPERIMENTAL SECTION}

Materials. 3-(Trimethylsilyl)-2,2,3,3-tetradeutero-propionic acid sodium salt (TSP, CAS N. 24493-21-8, 99 \%D, Armar Chemicals, Döttingen, Switzerland), sodium azide (NaN3, CAS N. 26628-22-8; $\geq 99.5 \%$, Sigma-Aldrich, Milan, Italy), deuterium oxide (D2O, CAS. N. 7789-20-0, $99.86 \%$ D, Eurisotop, Saclay, France) and methanol-d4 (CD3OD, CAS. N. 811-98-3, 99.80\%D, Eurisotop, Saclay, France) were used for sample preparation. NMR tubes (Norell 509-UP 7) were provided by Norell, Landisville NJ, US. The NMR samples were prepared using the automated system for liquid handling (SamplePro Tube, Bruker BioSpin).

Wine grape samples ( $c v$. Primitivo; Centro di Ricerca, Sperimentazione e Formazione in Agricoltura "Basile-Caramia (CRSFA), Locorotondo, Bari, Italy) were collected according to official recommendations (Regulations (CE) n. 834/2007, n. 889/2008, n. 1235/2008 and following modifications). 50 samples of $c v$. Primitivo were collected as follows: 30 berries were harvested randomly from different parts of the same plant for each sample. The samples were labelled according to the plant of origin, which was marked with a number and a letter, indicating respectively the vine-row and the sector of the vine-row to which the plant belonged. 1 bigger samples $(1 \mathrm{Kg})$ was collected randomly from 3 plants belonging to the vineyard and labelled according to the same procedure. The samples were refrigerated at $4{ }^{\circ} \mathrm{C}$ and transferred from the field to the laboratory, where they were stored at $-20^{\circ} \mathrm{C}$.

Experimental procedure. The interlaboratory comparison was organized according to EN ISO/IEC 17043:2010 and reference normative therein (Conformity assessment - General requirements for proficiency testing)[26] with 52 registered participants, 76 available spectrometers of which 65 producing results spectrometers [300, 400, 500, 600 and $700 \mathrm{MHz}$; Bruker (52), Agilent (9) and Jeol (4) manufacturers].[27] 75 sets of 8 NMR tubes were delivered to the participants and 65 spectrometers returned NMR data. The ILC participants were furnished with eight test NMR tubes, labelled as T, A, B, C, D, E, X, including a sample containing $c v$. Primitivo (tube $\mathrm{X}$ ), and five test tubes (A - E) containing spiked solutions of four metabolites naturally contained in the grape juice (glucose, fructose, arginine and alanine). Tube T contained pure methanol- $d_{4}\left(\mathrm{CD}_{3} \mathrm{OD}, 99.80 \% \mathrm{D}\right)$ and was used as an NMR thermometer to calibrate the temperature of each spectrometer at $298.1 \pm 0.1$ K.[28] Tube X, containing aqueous solutions of wine grape juice ( $c v$. Primitivo), was prepared as follows: 10 berries were defrosted at room temperature for 60 minutes. They were mechanically pressed and the resulting grape juice $(\sim 5 \mathrm{ml})$ was centrifuged (Ettich Rotofix 32A, $2500 \mathrm{~g}, 15$ minutes). The supernatant (1.08 ml) was combined with a solution $(84.6 \mathrm{mg} / 50 \mathrm{ml})$ of $\mathrm{NaN}_{3}$ in buffer $\left[\left(\mathrm{HC}_{2} \mathrm{O}_{4}\right)^{-} /\left(\mathrm{C}_{2} \mathrm{O}_{4}\right)_{2}{ }^{-} 0.11 \mathrm{M}\right.$, pH 4.2], giving Solution M1. $318 \mu 1$ of this solution was combined stepwise with a volume of the buffer solution $(222 \mu \mathrm{l})$ and a volume of a TSP/D $/ \mathrm{D}_{2} \mathrm{O}$ solution $(60 \mu \mathrm{L}, 0.10 \mathrm{~g}$ of 
TSP in $50 \mathrm{~g}$ of $\mathrm{D}_{2} \mathrm{O}$ ). To reach the final levels of metabolites concentrations in the tubes $\mathrm{A}, \mathrm{B}, \mathrm{C}, \mathrm{D}$ and $\mathrm{E}$ (figure 2), portions of the solution $\mathrm{M} 1$ prepared on big scale for the preparation of tube $\mathrm{X}$ were combined opportunely with the following two mixtures of metabolites in the buffer $\left[\left(\mathrm{HC}_{2} \mathrm{O}_{4}\right)^{-} /\left(\mathrm{C}_{2} \mathrm{O}_{4}\right)_{2}^{-} 0.11 \mathrm{M}\right.$, pH 4.2]: solution $\mathrm{M} 2$ was composed of glucose $\left(8.3 \cdot 10^{-1} \mathrm{M}\right)$ and arginine $\left(1.43 \cdot 10^{-3} \mathrm{M}\right)$; solution $\mathrm{M} 3$ was composed of fructose $\left(8.3 \cdot 10^{-2} \mathrm{M}\right)$ and alanine $\left(1.68 \cdot 10^{-4} \mathrm{M}\right)$.

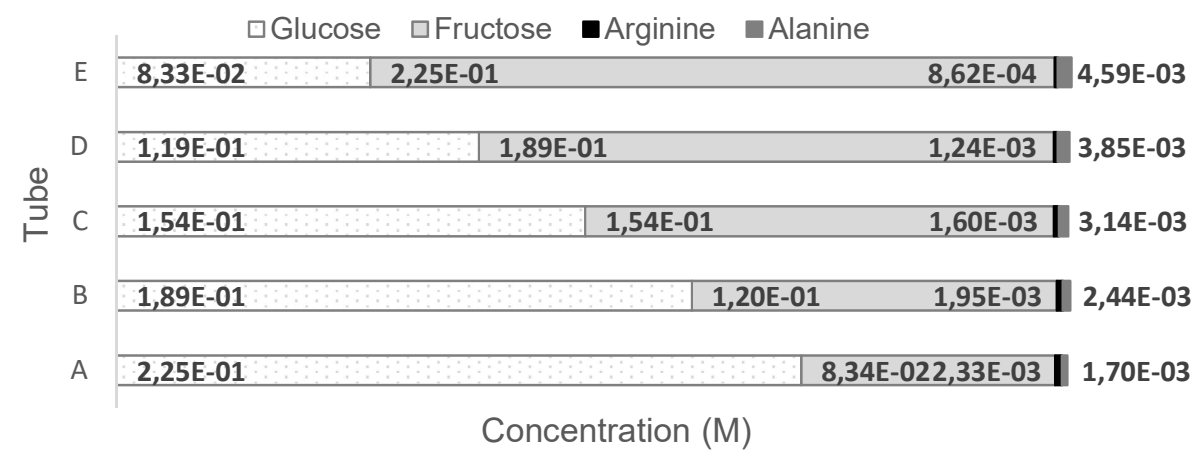

Figure 2. The final five levels of metabolites concentrations reached in the spiked solutions which are contained in tubes $\mathrm{A}-\mathrm{E}$.

Data acquisition and processing. For each sample the participants were asked to perform five repetitions of a $1 \mathrm{D}{ }^{1} \mathrm{H}$ NOESY NMR experiment,[29] preceded by a selective pre-saturation step to remove the residual water signal. The 5-fold replication was needed to comply with conditions for intermediate precision, i.e. same NMR tube, same spectrometer, same user, at least $24 \mathrm{~h}$ delay between runs, removal of the NMR tube from the magnet from run to run. The participants received experimental instructions for setting the acquisition parameters according to the spectrometer manufacturer requirements.

For Varian/Agilent spectrometers, guidelines included: pulse program (NOESY); size of fid (np, $128 \mathrm{~K}$ ); spectral width (sw, 20 ppm); transmitter offset (tof): ca. $4.70 \mathrm{ppm}$ (set the chemical shift value on the residual water signal); $90^{\circ}$ hard pulse (pw, optimized by manual or automatic procedures keeping the pulse length as short as possible, preferably $<10 \mu \mathrm{s}$, if hardware allows it); steady state (ss, 8); number of transients (nt, 64); mixing time (mixN, $0.01 \mathrm{~s})$; recycle delay (d1, $5 \mathrm{~s})$; no sspul (sspul = 'n'); no ZQ filter $($ Gzqfilt $=$ 'n'); no homo spoil during mixing time $($ gtl $=0$, gzlvl1 $=0$ and gstab $=0)$; presaturation during the whole length of d1, centered at the HDO residual signal with a nutation frequency of about $25 \mathrm{~Hz}$ [satmode = 'yn', satdly $=\mathrm{d} 1$, satfrq = tof; satpwr should be set to yield $\mathrm{r} 1$ of about 25 after running the command getpower(satpwr,tn):r1]; receiver gain optimization (once optimized for tube $\mathrm{A}$, use the obtained receiver gain value also for all replicates and for all tubes $\mathrm{A}-\mathrm{E}, \mathrm{X}$ and $\mathrm{Y}$ ).

For Bruker spectrometers, guidelines included: pulse program: noesyprld; size of FID (TD, $128 \mathrm{~K}$ ); spectral width (SW, 20 ppm); transmitter offset, ca. $4.70 \mathrm{ppm}$ (set at the chemical shift value of the residual water signal); $90^{\circ}$ hard pulse (p1, optimized by manual or automatic procedures keeping the pulse length as short as possible, preferably $<10 \mu \mathrm{s}$, if hardware allows it); power level for presaturation (p19, calculated by command "pulse 25Hz" after optimization of p1); dummy scans (ds, 8); number of scans (ns, 64); mixing time $(\mathrm{d} 8,0.01 \mathrm{~s})$; recycle delay $(\mathrm{d} 1,5 \mathrm{~s})$; receiver gain optimization (once optimized for tube $\mathrm{A}$, use the obtained receiver gain value also for all replicates and for all tubes A - E, X).

For Jeol spectrometers, guidelines included: pulse program: noesy abs; y points $=1$; size of fid $(\mathrm{x}$ point $=131072)$; spectral width (x_sweep $=20)$; transmitter offset (x_offset $=4.7) ; 90^{\circ}$ hard pulse $\left(x \_p u l s e=x 90 ; x \_\right.$atn $\left.=x a t n\right)$ to be optimized by manual or automatic procedures, keeping pulse length as short as possible, preferably $<10 \mu \mathrm{s}$; steady state (x_prescans $=8$ ); number of transients $($ scans $=64)$; mixing time $($ mix_time $=0.01)$; recycle delay (relaxation_delay $=5)$; presaturation during the whole length of recycle delay, centered at the HDO residual signal with a $\gamma \mathrm{B}_{2}$ power of about $25 \mathrm{~Hz}$ (irr_mode $=$ presaturation; irr-offset $=\mathrm{x} \_$offset; presat time flag $=\mathrm{y}$ ); use the following formula to calculate the value of irr attenuator corresponding to $25 \mathrm{~Hz}$ : irr attenuation $=\mathrm{x}$ atn $+20 \log (10.000 / x 90)$; receiver gain optimization (once optimized for tube A, use the obtained receiver gain value also for all replicates and for all tubes B-E, X).

The NMR raw data sets (FIDs and signal integrals) were uploaded by each laboratory on the website http://nmr.mxcs.it/index.php developed according to internationally agreed procedures.[30,31] The NMR spectra could be re-processed Topspin 1.3 - AMIX 3.9.9 (Bruker BioSpin GmbH, Germany) and Mnova (Mestrelab Research, Spain). FID was zero-filled with 128 K number of points. FIDs were extended by zero-filling to a final size of $256 \mathrm{~K}$. Fourier transformation was performed by applying an exponential multiplication function with a line broadening of $0.1 \mathrm{~Hz}$. Users could choose to apply either manual or automatic procedures for the phase and the baseline correction (without any limitations provided that the same procedure is applied to all NMR spectra). The calculation of peak area was the only procedure accepted for the signal integration. The TSP singlet signal $(0.00 \mathrm{ppm})$ was used as internal reference compound, upon ascertaining it was stable in solution over the time (see supplementary data for further details).

\section{RESULTS AND DISCUSSION}

Data analysis focused on the NMR signals related to alanine (S1), arginine (S2), glucose (S3, S6, and S7) and fructose (S4 and S5) as shown in Figure 3. The signal intensities were calculated as the areas subtending the signals in the following regions: $[1.42 \div 1.51$

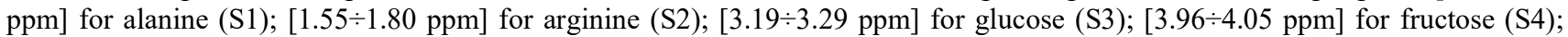
$[4.06 \div 4.15 \mathrm{ppm}]$ for fructose (S5); $[4.58 \div 4.71 \mathrm{ppm}]$ for glucose (S6); $[5.15 \div 5.31 \mathrm{ppm}]$ for glucose (S7). Each signal intensity was 
scaled to that of TSP calculated in the range $[-0.10 \div 0.10 \mathrm{ppm}]$. The width of the integration intervals was opportunely chosen to overcome the small changes in the position and in the shape of the signals unavoidably occurring when the spectra are produced at different field strengths (see "Selection of spectra regions for signals integration" in supplementary data for further details). Besides, the interest towards alanine and arginine derived from the fact that these compounds are among the best yeast nitrogen sources during alcoholic and malolactic fermentation in wine production.[32] Moreover, their NMR signals offer the opportunity to evaluate the reproducibility of weak signal intensities. Glucose and fructose were considered because of the importance of their quantification in wine grapes. Compared to other analytical techniques, NMR spectroscopy offers the advantage to identify and quantify glucose and fructose without any preventing separation procedure. Five signals (S3, S6 and S7 belonging to glucose and S4 and S5 belonging to fructose) were considered not only with the aim to quantify the two sugars but also to evaluate the critical issues deriving from proximity of the radiofrequency offset of the pre-saturation pulses to the signal frequencies.

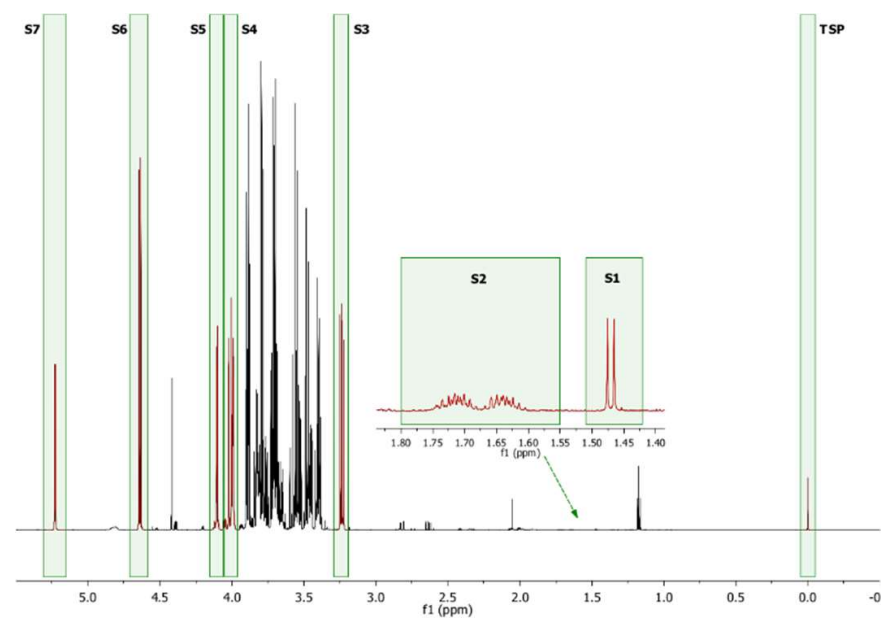

Figure 3. Typical 1D ${ }^{1} \mathrm{H}-\mathrm{NOESY}$ spectrum $\left(700 \mathrm{MHz}, \mathrm{D}_{2} \mathrm{O}\right.$ ) of NMR tube $\mathrm{X}$ containing an unspiked solution of grape juice ( $c v$. Primitivo).

A total number of 65 regression lines, one per spectrometer, were developed for each selected signal by using the $\mathrm{I}_{\text {signal }} / \mathrm{I}_{\mathrm{TSP}}$ ratios ( $\mathrm{I}_{\text {signal }}$ refers to the integral of the selected regions $\mathrm{S} 1-\mathrm{S} 7$ of the spectrum and $\mathrm{I}_{\mathrm{TSP}}$ refers to the integral of the internal reference compound) calculated taking into account the five test tubes $(\mathrm{A}-\mathrm{E})$ containing the spiked juice solutions and the tube $\mathrm{X}$ containing the unspiked grape juice sample. Each tube was submitted to 5-fold replicated experiment. The 65 regression lines were subjected to five selection tests aimed to remove anomalous data and to select the suitable lines allowing for the development of the most performing regression models able to calculate the concentration of the selected metabolite. The first test (T1 - linearity) was applied to evaluate the linearity between the dependent variable $y$ ( $\mathrm{I}_{\text {signal }} / \mathrm{I}_{\mathrm{TSP}}$ values) and the independent variable $x$ (spiked concentrations in tubes $\mathrm{A}-\mathrm{E}$ and original concentration in tube X). Only the regression lines passing T1 by mean of the F-statistic, were admitted to the second test (T2 - slope distribution) to identify and reject the lines having slopes which were recognized as outliers by Huber and Cochran tests. In turn, the lines passing T2 were submitted to a similar procedure (T3-y-intercept distribution) applied to the $y$ intercepts. All the variables belonging to the lines successfully passing T1, T2 and T3 were used to design a regression line which acted as a community-built reference line during the further two selection tests: T4 to select parallel lines (T4 - parallelism) and T5 to select coincident lines (T5 - coincidence).

As proof of concept, the following description refers to the selection process applied to S1 (alanine, $1.42 \div 1.51 \mathrm{ppm}$ ). The results obtained from the study of the other signals are reported in the supplementary data. According to the procedure described above, the 65 regression lines were submitted to the selection tests T1-T5. The results are shown in Figure 4 where the red lines are those failing the selection tests and the grey lines are those successfully passing the tests. 

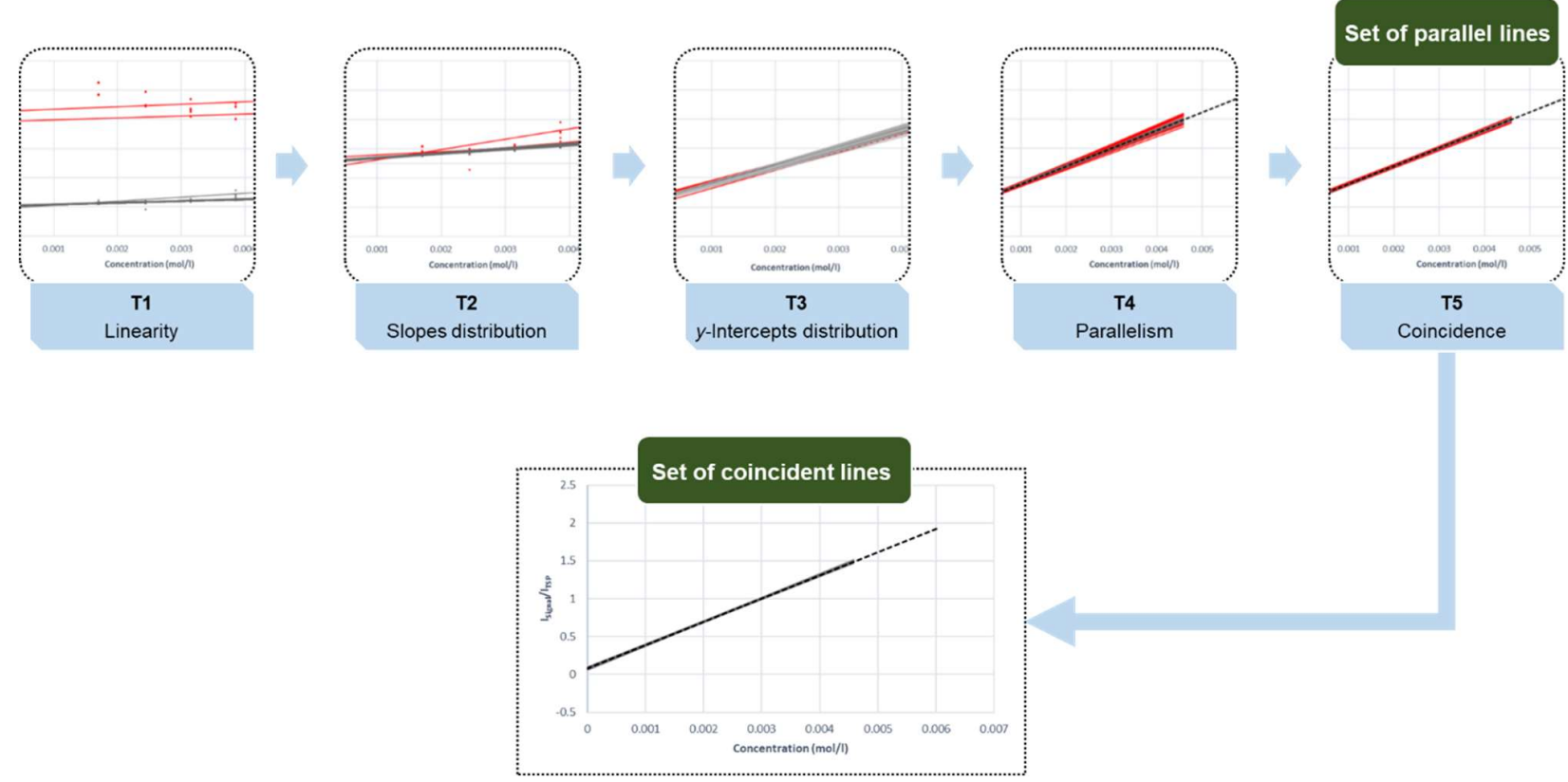

Figure 4. Plots of the regression lines produced for the signal S1 during the sequence of selection tests: a. T1-linearity test; b. T2-slopes distribution (Huber and Cochran tests on slopes); c. T3-y-intercepts distribution (Huber and Cochran tests on $y$-intercepts); d. T4-parallelism test; e. T5-coincidence test. Black lines passed successfully the selection tests; red lines failed the selection tests; black dashed line represents the community-built reference regression line.

T1 identified 2 lines (red lines in T1-linearity, figure 4) which resulted not linear and, thus, were excluded during the subsequent steps. Considering the slopes of the remaining 63 regression lines, T2 identified 4 outliers (red lines in T2-Slopes distribution, figure 4). The corresponding regression lines were removed from the data set. When the analogous test was applied to $y$-intercepts (T3), further 5 lines were rejected (red lines in T3-y-Intercepts distribution, figure 4). The resulting set of calibration data was used to develop a new regression line which acted as a community-built reference line (figure 4, dashed line) during the T4 and T5[33] to test the parallelism and the coincidence of each selected line with respect to the community-built reference line (see supplementary data for further details).

As a result of the described selection process, starting from initial 65 regression lines (ILC participants), only a reduced number of them passed successfully the sequence of T1-T5 tests, and could be deemed eligible for the quantification of the metabolites represented by the selected signals in the NMR spectrum (table 2).

Table 2. Number of regression lines produced by the ILC participants for the signals S1 - S7 which succeeded the sequence of chemometric tests assessing their proficiency for quantification purposes.

\begin{tabular}{l|l|lllllll}
\cline { 2 - 8 } & \multicolumn{1}{c|}{ Test } & S1 & S2 & S3 & S4 & S5 & S6 & S7 \\
\hline Entry 1 & T1-Linearity & 63 & 65 & 62 & 65 & 63 & 64 & 59 \\
Entry 2 & T2-Slope distribution & 59 & 53 & 59 & 62 & 60 & 55 & 55 \\
Entry 3 & T3-y-Intercept distribution & 54 & 49 & 58 & 59 & 56 & 54 & 54 \\
Entry 4 & T4-Parallelism & 34 & 49 & 52 & 54 & 51 & 52 & 48 \\
Entry 5 & T5-Coincidence & 25 & 20 & 17 & 22 & 18 & 9 & 14 \\
\hline
\end{tabular}

As summarized in table 2, the number of lines with good level of linearity (59 to 65 , table 2, entry 1 ) was high for all the seven selected signals, confirming that the nuclei response is directly proportional to their concentration with an excellent linearity between the independent variable (metabolite concentration) and the dependent one (area subtending the signal). In addition, the number of the lines remaining acceptable after the identification of the outliers among the slopes and the $y$-intercepts ( 49 to 59 , table 2 , entry 3 ) was satisfactory. Such data further confirmed the capability of the NMR spectroscopy to produce comparable lines, regardless the variability in the spectrometer features (see also supplementary data, tables S1-S7). Among the most demanding tests T4 and T5, T4 indicated that more than $50 \%$ of the lines ( 34 to 54 , table 2 , entry 4 ) could be safely considered parallel for all the selected signals. T5 behaved noticeably as a bottle neck during the selection process for all the signals and caused a dramatic decrease in the number of regression lines which resulted coincident ( 9 to 25 , table 2, entry 5) and thus, in principle, suitable for quantification. This behaviour was particularly pronounced for the signal S6 and this can be ascribed to its proximity to the pre-saturation offset. Indeed, differences in the calibration of the power level used for pre-saturation, depending on both the hardware features and the skills of the operator, may enhance the random error associated to the intensity of the signals close to the pre-saturation frequency.

Since the T5 - coincidence test caused a dramatic reduction of the regression lines which, in principle, were suitable for quantification, it was evaluated if satisfactory results in terms of reproducibility of concentration values were conditional on passing the 
highly demanding T5 test. Thus, the reproducibility of the data obtained by analysis of the parallel regression lines was compared with that one deriving from the analysis of coincident regression lines. In order to ascertain the suitability of the parallel lines passing $\mathrm{T} 4$ for a satisfactory quantification, the regression lines which resulted well-performing according to the test sequences T1-T4 and T1-T5 were employed to calculate the concentration of the metabolites represented by the signals S1-S7. The metabolite concentration in the tube $\mathrm{X}$ was estimated as $\mathrm{C}=-\mathrm{x}_{\text {intercept }}$, where $\mathrm{x}_{\text {intercept }}=-\mathrm{b} / \mathrm{a}$ derived from the regression lines of general formula $\mathrm{y}=$ $\mathrm{ax}+\mathrm{b}$ after setting null values for $y$. The predicted values were then subjected to the Huber and Cochran tests for identification of possible outliers which were removed from the further considerations. The performance of the spectrometers was evaluated according to their z-score, as $z=\left(C_{i}-C_{M}\right) / \sigma$ where $C_{i}$ represented the concentration predicted by each selected regression line, $C_{M}$ represented the average predicted concentration by the set of all the selected regression models, and $\sigma$ was the standard deviation calculated considering the corresponding predicted values (see supported material for further details).

Finally, the seven community-built reference lines were examined to get the values of the corresponding reference $x$-intercepts, which gave predicted reference concentrations $\left(C_{R}\right)$ for the seven selected metabolites. Since $C_{R}$ values were, in all cases, included in the "Horwitz region" $\left(1.2 \cdot 10^{-7} \leq \mathrm{C}_{\mathrm{R}} \leq 0.138\right)$, for each signal the predicted relative standard deviation (PRSD $\%$ ) was calculated by application of the Horwitz equation as PRSD $\%=2^{(1-\log R)}$ (where $C_{R}$ was expressed as mass/mass unit). The relative standard deviation (RSD\%) of the predicted concentration was calculated as RSD $\%=\sigma / C_{M} \times 100$, where $\sigma$ represented the standard deviation of the selected predicted values and $\mathrm{C}_{M}$ was the average of such values. The Horwitz ratio (HorRat), which is an accepted index assessing the data reproducibility in the context of interlaboratory comparisons, was evaluated as HorRat $=$ RSD\%/PRSD\%. Such ratio, under reproducibility conditions, should be a value between 0.5 and 2.[34] Not surprisingly, the parallel regression curves gave RSD\% values and, consequently, corresponding Horwitz ratios higher than those obtained from the coincident lines. Importantly, while the HorRat values for coincident lines (HorRat ${ }_{\mathrm{C}}$ ) were lower than 2 for all the seven signals, which could be defined as statistically equivalent, the HorRat values for parallel lines (HorRatP) were higher than 2, and, thus, unsatisfactory, only for S2 and S6. As a result of this study, it was found that though the introduction of the coincidence test was important to obtain high reproducibility for all the selected metabolites independently on the considered signal, passing the T4 - parallelism test was sufficient to get satisfactory reproducibility for all the signals but S2 and S6. It was ascertained (Tables S8, supplementary data) that such results were not related to spectrometer features (manufacturer, magnetic field strength, hardware configuration, year of fabrication).

Table 3. Average concentrations $\left(\mathrm{C}_{\mathrm{M}}\right.$ and $\left.\mathrm{C}^{\prime}{ }_{\mathrm{M}}\right)$ including the confidence interval predicted for the seven considered signals respectively by the selected coincident $\left(\mathrm{n}_{\mathrm{C}}\right)$ and parallel $\left(\mathrm{n}_{\mathrm{P}}\right)$ regression curves. The reproducibility of the predicted average concentrations was evaluated by mean of the Horwitz ratios $\left(\right.$ HorRat $_{C}$ and HorRat ${ }_{P}$ respectively for the case of the coincident curves and the parallel ones). $C_{R}(g / g)$, reference concentrations calculated according to the reference regression curve and included in the Horwitz equation. $\mathrm{n}$, number of coincident and parallel regression curves, respectively, for each signal. PRSD, predicted relative standard deviation calculated according to the Horwitz equation. $\mathrm{RSD}_{\mathrm{C}}$ and $\mathrm{RSD}_{\mathrm{P}}$, relative standard deviation of the metabolites concentration which were predicted by the coincident regression curves and by the parallel ones, respectively.

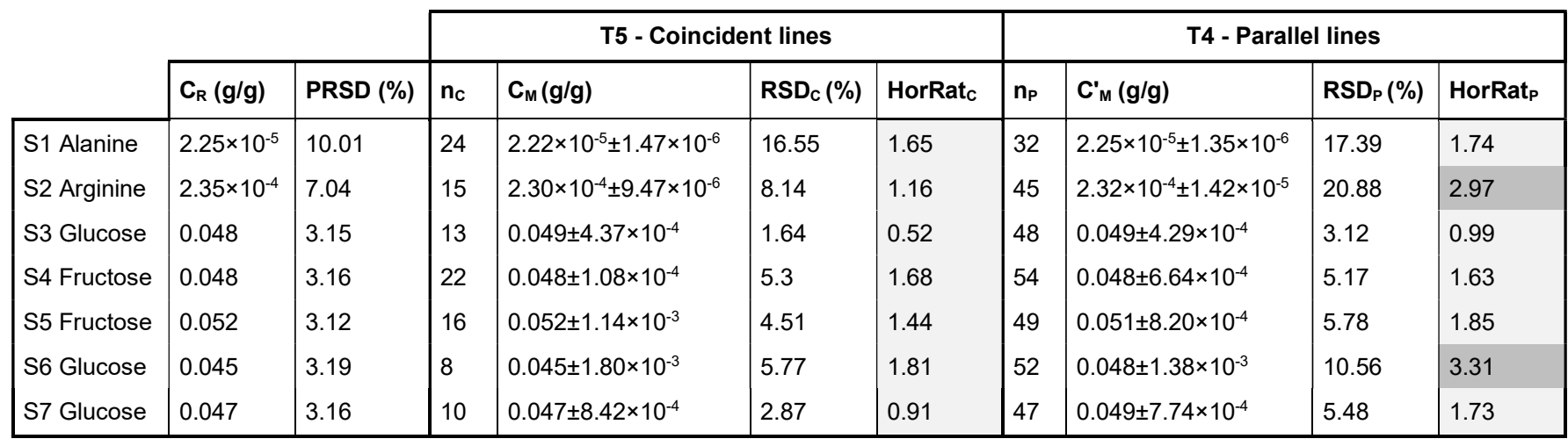

\section{CONCLUSION}

We explored the exclusive advantage offered by NMR spectroscopy to produce, for a given sample analysed by different spectrometers, a same instrumental response with high reproducibility. Such peculiarity, that is the independence of the instrumental response from magnetic field strength, hardware configuration, manufacturer and age, was opportunely exploited to develop a community-built calibration system able to furnish a reference calibration line along with a reference predicted concentration. The calibration data produced by 65 different spectrometers involved in an interlaboratory comparison were analysed and only the calibration lines passing T1-T3 tests were admitted to the development of the community-built reference calibration line. Therefore, the lines which resulted parallel and coincident with respect to the newly built reference line could be enrolled in the community-built calibration system, which was, ultimately, employed to quantify the analytes. The reproducibility of the predicted concentrations resulted highly satisfactory for all the selected metabolites, even when the set of parallel lines was the only one employed.

Many perspectives come from the present study opening to further development of this analytical approach, which should give rise, ideally, to a database containing a number of community-built reference calibration lines for a range of analytes. Importantly, such database may be continuously updated thanks to the contribution of external calibration lines which must be built under the same experimental conditions and, upon passing the described sequence of chemometric tests, must result suitable to be enrolled in 
the community-built calibration system. Consequently, the community-built reference line, taking advantage from the additional calibration data furnished by the external laboratories should become progressively more accurate and precise, thus paving the way to an open interlaboratory comparison. The up to date calibration system may be advantageously used by the local laboratories to evaluate their performance during the qNMR analysis of a given analyte.

This work may enable a more extensive use of qNMR method in standardized analytical protocols, overcoming the lack of official guidelines assessing the performance of qNMR analyses which still hampers the use of NMR methods as internationally accepted analytical protocols.

\section{ACKNOWLEDGMENT}

This work was supported by Programma Sviluppo Rurale FEASR 2007 -2013 Mis. 214 Az. 4 sub az. a) "Progetti integrati per la Biodiversità" Reg. (CE) 1698/2005 and Programma Sviluppo Rurale FEASR 2014-2020 Mis.10.2.1 "Progetti per la conservazione e valorizzazione delle risorse genetiche in agricoltura" Reg. (CE) 1305/2013. The authors are thankful to Iola Duarte from Universidade de Aveiro (CICECO) Portugal for her constructive suggestions and comments.

\section{REFERENCES}

[1] JCGM, JCGM 200:2008 International vocabulary of metrology-Basic and general concepts and associated terms (VIM) Vocabulaire international de métrologie-Concepts fondamentaux et généraux et termes associés (VIM), 2008. www.bipm.org.

[2] P. de Bièvre, R. Dybkaer, A. Fajgelj, D.B. Hibbert, Metrological traceability of measurement results in chemistry: Concepts and implementation (IUPAC Technical report), Pure Appl. Chem. 83 (2011) 1873-1935. https://doi.org/10.1351/PAC-REP-07-09-39.

[3] V. Gallo, R. Ragone, B. Musio, S. Todisco, A. Rizzuti, P. Mastrorilli, S. Pontrelli, N. Intini, P. Scapicchio, M. Triggiani, A. Pascazio, C. Cobas, S Mari, C. Garino, M. Arlorio, D. Acquotti, C. Airoldi, F. Arnesano, M. Assfalg, A. Barison, F. Benevelli, A. Borioni, L.R. Cagliani, L. Casadei, F.C. Marincola, K. Colson, R. Consonni, G. Costantino, M.A. Cremonini, S. Davalli, I. Duarte, S. Guyader, E. Hamon, M. Hegmanns, R. Lamanna, F. Longobardi, D. Mallamace, S. Mammi, M. Markus, L.R.A. Menezes, S. Milone, D. Molero-Vilchez, A. Mucci, C. Napoli, M.C. Rossi, E. SáezBarajas, F. Savorani, E. Schievano, F. Sciubba, A. Sobolev, P.G. Takis, F. Thomas, P. Villa-Valverde, M. Latronico, A Contribution to the Harmonization of Non-targeted NMR Methods for Data-Driven Food Authenticity Assessment, Food Anal. Methods. (2019). https://doi.org/10.1007/s12161-019-01664-8.

[4] V. Gallo, N. Intini, P. Mastrorilli, M. Latronico, P. Scapicchio, M. Triggiani, V. Bevilacqua, P. Fanizzi, D. Acquotti, C. Airoldi, F. Arnesano, M. Assfalg, F. Benevelli, D. Bertelli, L.R. Cagliani, L. Casadei, F. Cesare Marincola, G. Colafemmina, R. Consonni, C. Cosentino, S. Davalli, S.A. De Pascali, V. D’Aiuto, A. Faccini, R. Gobetto, R. Lamanna, F. Liguori, F. Longobardi, D. Mallamace, P. Mazzei, I. Menegazzo, S. Milone, A. Mucci, C. Napoli, T. Pertinhez, A. Rizzuti, L. Rocchigiani, E. Schievano, F. Sciubba, A. Sobolev, L. Tenori, M. Valerio, Performance Assessment in Fingerprinting and Multi Component Quantitative NMR Analyses, Anal. Chem. 87 (2015) 6709-6717. https://doi.org/10.1021/acs.analchem.5b00919.

[5] N.E. Jacobsen, NMR spectroscopy explained : simplified theory, applications and examples for organic chemistry and structural biology, WileyInterscience, 2007. https://books.google.it/books?id=KCkiiQ0uefoC\&printsec=frontcover\&dq=nmr+theory\&hl=en\&sa=X\&ved=0ahUKEwjzgJCUv9fiAhUEjqQKHc

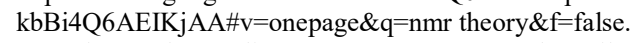

[6] J. Keeler, Understanding NMR spectroscopy, John Wiley and Sons, 2010.

[7] S.K. Bharti, R. Roy, Quantitative 1H NMR spectroscopy, TrAC - Trends Anal. Chem. 35 (2012) 5-26. https://doi.org/10.1016/j.trac.2012.02.007.

[8] K. Chen, Mehdi Mobli and Jeffrey C. Hoch (Eds.): Fast NMR data acquisition: beyond the Fourier transform, Anal. Bioanal. Chem. 410 (2018) 1615-1616. https://doi.org/10.1007/s00216-017-0846-0.

[9] T. Parella, Towards perfect NMR: Spin-echo versus perfect-echo building blocks, Magn. Reson. Chem. 57 (2019) 13-29. https://doi.org/10.1002/mrc.4776.

[10] $\bar{E}$. Kupče, T.D.W. Claridge, Molecular structure from a single NMR supersequence, Chem. Commun. 54 (2018) $7139-7142$. https://doi.org/10.1039/C8CC03296C.

[11] M. Foroozandeh, R.W. Adams, N.J. Meharry, D. Jeannerat, M. Nilsson, G.A. Morris, Ultrahigh-Resolution NMR Spectroscopy, Angew. Chemie Int. Ed. 53 (2014) 6990-6992. https://doi.org/10.1002/anie.201404111.

[12] D. Raftery, High-throughput NMR spectroscopy, Anal. Bioanal. Chem. 378 (2004) 1403-1404. https://doi.org/10.1007/s00216-003-2437-5.

[13] I.C. Felli, B. Brutscher, Recent Advances in Solution NMR: Fast Methods and Heteronuclear Direct Detection, ChemPhysChem. 10 (2009) 13561368. https://doi.org/10.1002/cphc.200900133.

[14] Y. Liu, J. Cheng, H. Liu, Y. Deng, J. Wang, F. Xu, NMRSpec: An integrated software package for processing and analyzing one dimensional nuclear magnetic resonance spectra, Chemom. Intell. Lab. Syst. 162 (2017) 142-148. https://doi.org/10.1016/J.CHEMOLAB.2017.01.005.

[15] A. Mohamed, C.H. Nguyen, H. Mamitsuka, NMRPro: an integrated web component for interactive processing and visualization of NMR spectra, Bioinformatics. 32 (2016) 2067-2068. https://doi.org/10.1093/bioinformatics/btw102.

[16] C. Cobas, I. Iglesias, F. Seoane, NMR data visualization, processing, and analysis on mobile devices, Magn. Reson. Chem. 53 (2015) $558-564$. https://doi.org/10.1002/mrc.4234.

[17] L. Castañar, G.D. Poggetto, A.A. Colbourne, G.A. Morris, M. Nilsson, The GNAT: A new tool for processing NMR data, Magn. Reson. Chem. 56 (2018) 546-558. https://doi.org/10.1002/mrc.4717.

[18] T. Schonberger, Y.B. Monakhova, D.W. Lachenmeier, T. Kuballa, EUROLABS Technical Report No. 01/ 2014 May 2014, Eurolab. (2014) 1-20. http://www.eurolab.org/documents/EUROLAB Technical Report NMR Method Development and Validation May 2014_final.pdf.

[19] U.S. Ellison Secretary, R. Bettencourt da Silva, E.P. Poland Fodor, R. Kaarls, E.B. Germany Magnusson, I.P. Robouch, E. St Gallen, S.A. van der Veen, M.W. Walsh Eurachem IRE Wegscheider, P. Yolci Omeroglu, E. Representatives, EURACHEM/CITAC Guide Quantifying Uncertainty in Analytical Measurement Composition of the Working Group, 2009. https://www.eurachem.org/images/stories/Guides/pdf/QUAM2012_P1.pdf.

[20] T. Schönberger, Y.B. Monakhova, D.W. Lachenmeier, S. Walch, T. Kuballa, N.-P.E. Team, (NEXT) -NMR working group Germany, EUROLAB Technical Report 01/2015 -Guide to NMR Method Development and Validation-Part II: Multivariate data analysis, Brussels, 2015. http://www.eurolab.org/documents/NMR Val Guideline II V6.pdf.

[21] T. Schönberger, Y.B. Monakhova, D.W. Lachenmeier, T. Kuballa, N.-P.E. Team, (NEXT) -NMR working group Germany, EUROLAB Technical Report 01/2014 - "Guide to NMR Method Development and Validation-Part 1: Identification and Quantification," Brussels, 2015. http://www.eurolab.org/documents/EUROLAB Technical Report NMR Method Development and Validation May 2014_final.pdf.

[22] S. Monsonis Centelles, H.C.J. Hoefsloot, B. Khakimov, P. Ebrahimi, M. V. Lind, M. Kristensen, N. de Roo, D.M. Jacobs, J. van Duynhoven, C. Cannet, F. Fang, E. Humpfer, H. Schäfer, M. Spraul, S.B. Engelsen, A.K. Smilde, Toward Reliable Lipoprotein Particle Predictions from NMR Spectra of Human Blood: An Interlaboratory Ring Test, Anal. Chem. 89 (2017) 8004-8012. https://doi.org/10.1021/acs.analchem.7b01329.

[23] J.L. Ward, J.M. Baker, S.J. Miller, C. Deborde, M. Maucourt, B. Biais, D. Rolin, A. Moing, S. Moco, J. Vervoort, A. Lommen, H. Schäfer, E. Humpfer, M.H. Beale, An inter-laboratory comparison demonstrates that [1H]-NMR metabolite fingerprinting is a robust technique for collaborative plant metabolomic data collection, Metabolomics. 6 (2010) 263-273. https://doi.org/10.1007/s11306-010-0200-4. 
[24] P. Giraudeau, I. Tea, G.S. Remaud, S. Akoka, Reference and normalization methods: Essential tools for the intercomparison of NMR spectra, J. Pharm. Biomed. Anal. 93 (2014) 3-16. https://doi.org/10.1016/J.JPBA.2013.07.020.

[25] M.R. Viant, D.W. Bearden, J.G. Bundy, I.W. Burton, T.W. Collette, D.R. Ekman, V. Ezernieks, T.K. Karakach, C.Y. Lin, S. Rochfort, J.S. de Ropp, Q. Teng, R.S. Tjeerdema, J.A. Walter, H. Wu, International NMR-Based Environmental Metabolomics Intercomparison Exercise, Environ. Sci. Technol. 43 (2009) 219-225. https://doi.org/10.1021/es802198z.

[26] ISO 17043-2010_CONFORMITY ASSESSMENT - GENERAL REQUIREMENTS FOR PROFICIENCY TESTING, n.d. https://www.iso.org/standard/29366.html.

[27] V. Gallo, N. Intini, P. Mastrorilli, M. Latronico, S. Todisco, A. Rizzuti, R. Ragone, P. Scapicchio, P. Dambruoso, M.A. Cremonini, F. Benevelli, S. Ghelli, NMR Inter-laboratory Comparisons: Validation of a 1D 1H-NOESY experiment for fingerprinting of grape juices, Rome, 2017.

[28] M. Findeisen, T. Brand, S. Berger, A1H-NMR thermometer suitable for cryoprobes, Magn. Reson. Chem. 45 (2007) 175-178. https://doi.org/10.1002/mrc.1941.

[29] R.T. Mckay, How the 1D-NOESY suppresses solvent signal in metabonomics NMR spectroscopy: An examination of the pulse sequence components and evolution, Concepts Magn. Reson. Part A. 38A (2011) 197-220. https://doi.org/10.1002/cmr.a.20223.

[30] International Organization for Standardization (ISO), ISO 13528: Statistical methods for use in proficiency testing, 2005 (2005) 76. https://www.iso.org/standard/35664.html.

[31] ISO, ISO 5725-1:1994 - Accuracy (trueness and precision) of measurement methods and results - Part 1: General principles and definitions, (2012) 17. https://www.iso.org/standard/11833.html.

[32] F. Zamora, Biochemistry of alcoholic fermentation, in: Wine Chem. Biochem., Springer New York, 2009: pp. 3-26. https://doi.org/10.1007/978-0387-74118-5_1.

[33] Statistical Analysis Methods for Chemists, Royal Society of Chemistry, 2007. https://doi.org/10.1039/9781847551924

[34] C. Rivera, R. Rodriguez, Horwitz equation as quality benchmark in ISO / IEC 17025 testing laboratory, IIE Annu. Conf. (2010). http://bii.mx/documentos/horwitzCf11.pdf. 